International Journal of Pure and Applied Mathematics

Volume 114 No. 3 2017, 639-646

ISSN: 1311-8080 (printed version); ISSN: 1314-3395 (on-line version)

url: http://www.ijpam.eu

doi: 10.12732/ijpam.v114i3.18

\title{
OSCILLATION CRITERIA FOR SECOND ORDER LINEAR GENERALIZED DIFFERENCE EQUATION
}

\author{
M. Maria Susai Manuel ${ }^{1}$, P. Venkata Mohan Reddy ${ }^{2}$ \\ ${ }^{1,2}$ Department of Mathematics \\ R.M.D. Engineering College \\ Kavaraipettai - 601 206, Tamil Nadu, S. INDIA
}

\begin{abstract}
In this paper, we obtain some oscillation criteria for the second order generalized linear difference equation

$$
\Delta_{\ell}^{2} u(k)+p(k) u(k)=0
$$

where $k \in[0, \infty), p \in L^{\prime}\left(\left[u_{0}, \infty\right)\right)$ and $\ell$ is a positive real.

AMS Subject Classification: 39A10, 39A11

Key Words: generalized difference equation, oscillation, non-oscillation

\section{Introduction}

The basic theory of difference equations is based on the operator $\Delta$ defined as $\Delta u(k)=u(k+1)-u(k), \quad k \in \mathbb{N}=\{0,1,2,3, \cdots\}$. Eventhough many authors [1] and [14]-[16] have suggested the definition of $\Delta$ as

$$
\Delta u(k)=u(k+\ell)-u(k), k \in \mathbb{R}, \ell \in \mathbb{R}-\{0\},
$$

no significant progress took place on this line. But recently, when we took up the definition of $\Delta$ as given in (2) we developed the theory of difference equations in a different direction ([8]-[9]). For convenience, we labelled the operator $\Delta$ defined by (2) as $\Delta_{\ell}$ and by defining its inverse $\Delta_{\ell}^{-1}$, many interesting results and applications in number theory were established (see [8], [10], [11], [12], [13]).

Received: April 16, 2017

Revised: $\quad$ April 29, 2017

Published: $\quad$ May 23, 2017

$\S_{\text {Correspondence author }}$ (c) 2017 Academic Publications, Ltd. url: www.acadpubl.eu 
In 1957, Z. Nehari [4] and Jiqin Deng [3] worked on Oscillation criteria for second order linear differential equations. In fact, in the last few years, several monographs and hundreds of papers have been written; see for example the monographs [see [1], [2]] and the papers [[5] - [7]]. But only very few papers are available on the qualitative properties of solutions of difference equations involving $\Delta_{\ell}$. To move forward on this area, in this paper, we study the oscillation property of the second order generalized linear difference equation (1).

Throughout this paper we use the following notations:

(i) $\mathbb{N}=\{0,1,2,3, \ldots\}, \mathbb{N}(a)=\{a, a+1, a+2, \ldots\}$,

(ii) $\mathbb{N}_{\ell}(j)=\{j, j+\ell, j+2 \ell, \ldots\}$,

(iii) $\lceil x\rceil$ upper integer part of $x$.

\section{Preliminaries}

Definition 1. [8] Let $u(k), k \in[0, \infty)$ be a real or complex valued function and $\ell \in(0, \infty)$. Then, the generalized difference operator $\Delta_{\ell}$ is defined as

$$
\Delta_{\ell} u(k)=u(k+\ell)-u(k) .
$$

Similarly, the generalized difference operator of the $r^{\text {th }}$ kind is defined as

$$
\Delta_{\ell}^{r} u(k)=\underbrace{\Delta_{\ell}\left(\Delta_{\ell}\left(\ldots\left(\Delta_{\ell} u(k)\right) \ldots\right)\right)}_{r \text { times }} .
$$

Definition 2. [8] Let $u(k), k \in[0, \infty)$ be a real or complex valued function and $\ell \in(0, \infty)$. Then, the inverse of $\Delta_{\ell}$ denoted by $\Delta_{\ell}^{-1}$ is defined as follows.

$$
\text { If } \Delta_{\ell} v(k)=u(k) \text {, then } v(k)=\Delta_{\ell}^{-1} u(k)+c_{j},
$$

where $c_{j}$ is a constant for all $k \in \mathbb{N}_{\ell}(j), j=k-\left[\frac{k}{\ell}\right] \ell$.

In general, $\Delta_{\ell}^{-n} u(k)=\Delta_{\ell}^{-1}\left(\Delta_{\ell}^{-(n-1)} u(k)\right)$ for $n \in \mathbb{N}(2)$.

Lemma 3. [8] If the real valued function $u(k)$ is defined for all $k \in[a, \infty)$, then

$$
\Delta_{\ell}^{-1} u(k)=\sum_{r=1}^{\left[\frac{k-a}{\ell}\right]} u(k-r \ell)+c_{j},
$$

where $c_{j}$ is a constant for all $k \in \mathbb{N}_{\ell}(j), j=k-a-\left[\frac{k-a}{\ell}\right] \ell$. 
Theorem 4. If $\Delta_{\ell} v(k)=u(k)$ for $k \in\left[k_{2}, \infty\right)$ and $j=k-k_{2}-\left[\frac{k-k_{2}}{\ell}\right] \ell$, then $v(k)-v\left(k_{2}+j\right)=\sum_{r=0}^{\left[\frac{k-k_{2}-j-\ell}{\ell}\right]} u\left(k_{2}+j+r \ell\right)$.

Lemma 5. [9]

$$
\begin{aligned}
\Delta_{\ell}\{u(k) v(k)\} & =u(k+\ell) \Delta_{\ell} v(k)+v(k) \Delta_{\ell} u(k) \\
& =v(k+\ell) \Delta_{\ell} u(k)+u(k) \Delta_{\ell} v(k), \forall k \in \mathbb{N}_{\ell}(a) .
\end{aligned}
$$

Definition 6. The solution $u(k)$ of (1) is called oscillatory if for any $k_{1} \in[a, \infty)$ there exists a $k_{2} \in \mathbb{N}_{\ell}\left(k_{1}\right)$ such that $u\left(k_{2}\right) u\left(k_{2}+\ell\right) \leq 0$. The difference equation itself is called oscillatory if all its solutions are oscillatory. If the solution $u(k)$ is not oscillatory, then it is said to be nonoscillatory (i.e. $u(k) u(k+\ell)>0$ for all $\left.k \in\left[k_{1}, \infty\right)\right)$.

\section{Main Results}

Theorem 7. If for large $k \in \mathbb{N}_{\ell}(a)$,

$$
\sum_{s=k}^{\infty} p(s) \geq \frac{\alpha_{0}}{k}
$$

where $\alpha_{0}>\frac{1}{4}$, then (1) is oscillatory.

Proof. Assume that (8) holds. Let us assume that $u(k)$ be a non oscillatory solution of (1). Without loss of generality we assume that $u(k)>0$ for all $k \geq k_{0}>0$. By (8) it is easy to see that there exists $k_{1} \geq k_{0}$ such that

$$
\sum_{s=k}^{\infty} p(s) \geq \frac{\alpha_{0}}{k} \quad \text { for } \quad k \geq k_{1}
$$

which yields that there exists an integer $n(k) \geq k$, such that

$$
\begin{gathered}
\sum_{s=k}^{\infty} p(s)-\sum_{s=k^{\prime}}^{\infty} p(s) \geq \frac{\alpha_{1}}{k} \quad \text { and } \quad \frac{\alpha_{1}^{2}}{k}-\frac{\alpha_{1}^{2}}{k^{\prime}} \geq \frac{\alpha^{2}}{k} \quad \text { for } \quad k^{\prime} \geq n(k) \\
\sum_{s=k}^{k^{\prime}} p(s) \geq \frac{\alpha_{1}}{k},
\end{gathered}
$$


where $\alpha_{0} \geq \alpha_{1} \geq \alpha>\frac{1}{4}$.

Let $v(k)=\frac{\Delta_{\ell} u(k)}{u(k)} \quad$ for $\quad k \geq k_{0}$,

$$
\begin{aligned}
\Delta_{\ell} u(k) & =v(k) u(k) \quad \text { for } \quad k \geq k_{0}, \quad \text { then } \\
\Delta_{\ell}^{2} u(k) & =v(k+\ell) \Delta_{\ell} u(k)+\Delta_{\ell} v(k) u(k) \\
& =\left[v(k+\ell) v(k)+\Delta_{\ell} v(k)\right] u(k) .
\end{aligned}
$$

Substituting this into (1), we get

$$
\left[\Delta_{\ell} v(k)+v(k+\ell) v(k)+p(k)\right]=0 \quad \text { for } \quad k \geq k_{0} .
$$

Summing (11) form $k$ to $k^{\prime}$, and by (10) we get

$$
v(k)-v\left(k^{\prime}\right)=\sum_{s=k}^{k^{\prime}} v(s+\ell) v(s)+\sum_{s=k}^{k^{\prime}} p(s) \geq 0
$$

for $k^{\prime} \geq n(k)$ and $k \geq k_{1}$. If there exists $k_{2} \geq k_{1}$, such that $v\left(k_{2}\right)<0$, then from (12) for $k \geq n\left(k_{2}\right), v(k)<0$. Therefore, $v(k)$ is eventually positive (or) eventually negative. If $v(k)$ is eventually negative, then there exists $k_{3} \geq k$ such that $v(k) \leq 0$ for $k \geq k_{3}$ and

$$
|v(k)| \geq \sum_{s=k_{3}}^{k} v(s+\ell) v(s)+\sum_{s=k_{3}}^{k} p(u) \quad \text { for } \quad k \geq n\left(k_{3}\right)
$$

which, with (10) yields

$$
|v(k)| \geq \sum_{s=k_{3}}^{k} v(s+\ell) v(s)+\sum_{s=k_{3}}^{k} p(s) \geq \sum_{s=k_{3}}^{k} v(s+\ell) v(s)+\frac{\alpha_{1}}{k_{3}}
$$

i.e., $\quad|v(k)| \geq \frac{\alpha_{1}}{k_{3}} \geq \frac{\alpha}{k} \quad$ for $\quad k \geq n\left(k_{3}\right)$.

Substituting this into (12), we obtain

$$
|v(k)| \geq \sum_{s=n\left(k_{3}\right)}^{k} \frac{\alpha_{1}^{2}}{(s+\ell) s}+\frac{\alpha_{1}}{n\left(k_{3}\right)} \geq \frac{\tau_{0}^{2}+\tau_{0}}{k}
$$

for $k \geq n\left(n\left(k_{3}\right)\right)$, where $\tau_{0}=\alpha>\frac{1}{4}$. 
If $v(k)$ is eventually positive, then there exists $k_{4} \geq k_{1}$ such that $v(k)>0$ for $k \geq k_{4}$, and from (10) and (12), we obtain

$$
v(k) \geq \sum_{s=k}^{k^{\prime}} v(s+\ell) v(s)+\sum_{s=k}^{k^{\prime}} p(s) \geq \frac{\alpha}{k} \text { for } k^{\prime} \geq n(k) \text { and } k \geq k_{4}
$$

Applying similar method as applied in getting (15), we obtain

$$
|v(k)| \geq \frac{\tau_{0}^{2}+\tau_{0}}{k} \quad \text { for } \quad k \geq k_{4}
$$

Setting $\tau_{i}=\tau_{i-1}^{2}+\tau_{0}, i=1,2,3, \ldots$ and taking $k_{5}=\max \left\{n\left(n\left(k_{3}\right)\right), k_{4}\right\}$ from (15) and (17), we get

$$
|v(k)| \geq \frac{\tau_{1}}{k} \quad \text { for } \quad k \geq k_{5}
$$

By induction, from (14) and (16), we can prove that

$$
|v(k)| \geq \frac{\tau_{i}}{k} \quad \text { for } \quad k \geq k_{5}, \quad i=1,2,3, \ldots
$$

It is easy to see that, $|v(k)|=\infty$ for $k \geq k_{5}$. This contradiction yields that the proof is complete.

Theorem 8. If for large $k \in \mathbb{N}_{\ell}(a)$,

$$
\sum_{s=k}^{\infty} p(s) \leq \frac{1}{4 k}
$$

then (1) has an eventually positive solution.

Proof. Take $k_{0}>0$ such that

$$
\sum_{s=k}^{\infty} p(s) \leq \frac{1}{4 k} \quad k \geq k_{0}
$$

Define

$$
\begin{aligned}
v_{0}(k) & =v_{1}(k)=\frac{1}{2 k} \quad \text { for } \quad k \geq k_{0} \\
v_{i+2}(k) & =\sum_{s=k}^{\infty} v_{i}(s) v_{i+1}(s+\ell)+\sum_{s=k}^{\infty} p(s) \quad \text { for } \quad k \geq k_{0} .
\end{aligned}
$$


which,together with (20) and (21), yield

$$
\begin{aligned}
v_{2}(k) & =\sum_{s=k}^{\infty} v_{0}(s) v_{1}(s+\ell)+\sum_{s=k}^{\infty} p(u) \\
& =\frac{1}{4} \sum_{s=k}^{\infty} \frac{1}{s(s+\ell)}+\sum_{s=k}^{\infty} p(s) \leq \frac{1}{4} \frac{1}{k}+\frac{1}{4 k}=\frac{1}{2 k}=v_{0}(k) .
\end{aligned}
$$

By induction, one can easily prove in-general that,

$$
\sum_{s=k}^{\infty} p(s) \leq v_{i+1}(k) \leq v_{i}(k) \leq \frac{1}{2 k} \quad \text { for } \quad k \geq k_{0}, i=0,1,2,3, \ldots
$$

Therefore, the sequence $\left\{v_{i}(k)\right\}$ has a limiting function $v(k)$ on $i$ with

$$
\sum_{s=k}^{\infty} p(s) \leq \lim _{i \rightarrow \infty} v_{i+1}(k)=v(k) \leq \frac{1}{2 k} \quad \text { for } \quad k \geq k_{0} .
$$

From (16), we find

$$
v(k)=\sum_{s=k}^{\infty} v(s+\ell) v(s)+\sum_{s=k}^{\infty} p(s) \quad \text { for } \quad k \geq k_{0} .
$$

Operating both sides on $\Delta_{\ell}$, it follows that

$$
\begin{gathered}
\Delta_{\ell} v(k)=\Delta_{\ell}\left[\sum_{s=k}^{\infty} v(s+\ell) v(s)+\sum_{s=k}^{\infty} p(s)\right]=-v(k+\ell) v(k)-p(k) . \\
\Delta_{\ell} v(k)+v(k+\ell) v(k)+p(k)=0 \text { for } \quad k \geq k_{0} .
\end{gathered}
$$

Set $u\left(k_{0}\right)=1, u(k)=u_{0} \prod_{r=1}^{\left\lceil\frac{k-a}{\ell}\right\rceil}[v(k-r \ell)+1]$ for $k \geq k_{0}$.

Then $u(k)>0, \Delta_{\ell} u(k)=v(k) u(k)$ for $k \geq k_{0}$. Hence,

$$
\begin{gathered}
\Delta_{\ell}^{2}(u(k))=v(k+\ell) \Delta_{\ell}(u(k))+\Delta_{\ell} v(k) u(k)=-p(k) u(k) \\
\Delta_{\ell}^{2} u(k)+p(k) u(k)=0 \quad \text { for } \quad k \geq k_{0} .
\end{gathered}
$$

Clearly, for $k \geq k_{0}, u(k)>0$, is a positive solution of (1).

The proof is completed. 
Example 9. The second order linear generalized difference equation $\Delta_{\ell}^{2} u(k)+\frac{3 \ell^{2}}{(4 k-\ell)(4 k+3 \ell)} u(k)=0$, and for $p(k)=\frac{3 \ell^{2}}{(4 k-\ell)(4 k+3 \ell)}$, satisfies Theorem 8 and hence it has a positive solution. In fact $u(k)=\prod_{r=1}^{\left\lceil\frac{k-\ell}{\ell}\right\rceil} \frac{4(k-r \ell)}{4(k-r \ell)-\ell}$ with $u(0)=1$ is a positive solution.

\section{References}

[1] R.P. Agarwal, Difference equations and inequalities, in: Theory, Methods and Applications, second ed., revised and expanded, Maarcel Dekker, New York (2000).

[2] R.P. Agarwal, P.J.Y. Wong, Advanced Topics in Difference Equations, Kluwer Academic Publishers, (1997).

[3] Jiqin Deng, Oscillation criteria for second order linear differential equation, J. Math. Anal. Appl., 271 (2002), 283-28\%.

[4] Z. Nehari, Oscillation criteria for second order linear differential equations, Tans, AMS, 85 (1957), 428-445.

[5] W. T. Patula, Growth and oscillation properties of second order linear difference equations, SIAM J. Math. Anal., 10(1979), 55-61.

[6] Sui Sun Cheng, Tze Cheung and Horng Jaan Li, Oscillation criteria for second order difference equation. Funkcialaj Ekvacioj, 34 (1991), 223-239.

[7] B. G. Zhang, G. D. Chen, Oscillation of certain second order nonlinear difference equation, J. Math. Anal. Appl., 199 (1996), 841-872.

[8] M.Maria Susai Manuel, G.Britto Antony Xavier and E.Thandapani, Theory of Generalized Difference Operator and Its Applications, Far East Journal of Mathematical Sciences, 20(2) (2006), 163-171.

[9] M.Maria Susai Manuel and G.Britto Antony Xavier, Generalized Difference calculus of sequences of real and complex numbers, International Journal of Computational and Numerical Analysis and applications, 6(4) (2004), 401-415.

[10] M.Maria Susai Manuel, Adem Kilicman, G.Britto Antony Xavier,R. Pugalarasu, D.S. Dilip, On the Solution of Second Order Generalized Difference Equations, Advances in Difference Equation, (2012) Article ID 105.

[11] Manuel M.M.S, Xavier GBA, Pugalarasu R, Dilip DS, An Application on the Second Order Generalized Difference Equations, Adv. Differ. Eq., (2013) Article ID 35.

[12] M.M.S. Manuel, Adem Kilicman, K. Srinivasan and G. Dominic Babu, Oscillation of Solutions of Some Generalized Non Linear $\alpha$-Difference Equations, Advances in Difference Equation, (2014), Article ID 109.

[13] M.Maria Susai Manuel, G.Britto Antony Xavier, V.Chandrasekar and R.Pugalarasu, On Generalized Difference Operator of Third Kind and its Applications to Number Theory, International Journal of Pure and Applied Mathematics, 53(1) (2009), 69-82.

[14] Ronald E.Mickens, Difference Equations, Van Nostrand Reinhold Company, New York (1990). 
[15] Saber N.Elaydi, An Introduction To Difference Equations, Second Edition, Springer, (1999).

[16] Walter G.Kelley, Allan C. Peterson, Difference Equations, An Introduction with Applications, Academic Press, inc (1991). 